\title{
Integrated photonic coupled Sagnac loop reflector filters for flat- top wavelength interleaving and switching
}

This paper was downloaded from TechRxiv (https://www.techrxiv.org).

\section{LICENSE}

CC BY 4.0

SUBMISSION DATE / POSTED DATE

23-08-2020 / 24-08-2020

\section{CITATION}

Moss, David (2020): Integrated photonic coupled Sagnac loop reflector filters for flat-top wavelength interleaving and switching. TechRxiv. Preprint. https://doi.org/10.36227/techrxiv.12846977.v1

$\mathrm{DOI}$ 


\title{
Integrated photonic coupled Sagnac loop reflector filters for flat-top wavelength interleaving and switching
}

\author{
Hamed Arianfard, Jiayang Wu, and David J. Moss* \\ Optical Sciences Centre, Swinburne University of Technology, Hawthorn, VIC 3122, Australia. \\ *E-mail:dmoss@swin.edu.au
}

\begin{abstract}
We investigate integrated photonic resonators formed by coupled Sagnac loop reflectors (CSLR). Mode splitting in the CSLR resonator is tailored to provide flat-top spectral response for wavelength interleaving and non-blocking switching functions in $\mathrm{C}$ band.
\end{abstract}

\section{Introduction}

Optical interleavers performing signal multiplexing / demultiplexing both at the transmitters and receivers as well as optical switching nodes performing signal routing among a set of input and output ports are core components in wavelength division multiplexing (WDM) optical communication systems [1,2]. The capability to realize these devices in the form of photonic integrated circuits will reap the great dividends including compact footprint, low energy consumption, high scalability and the potential to significantly reduce cost by mass production [3, 4]. In WDM systems, optical interleavers and switching nodes with a flat-top spectral response that can minimize filtering distortions are highly desirable. To date, various schemes have been demonstrated to realize integrated optical interleavers and switching nodes [2, 5-8]. However, these devices, based on either finite-impulse-response (FIR) filters such as Mach-Zehnder interferometers (MZIs) or infiniteimpulse-response (IIR) filters such as Fabry-Perot (FP) or Gires-Tournois (GT) [9-13] cavities and microring resonators, suffer from a fundamental limitation in terms of filter shape that allows them to achieve flat-top spectral responses only by cascading many subunits. This imposes stringent requirements for the alignment of resonant wavelengths from separate subunits and limits the practical applications out of laboratory.

Recently [14, 15], we reported devices in silicon-on-insulator (SOI) nanowires formed by Sagnac loop reflectors (SLRs). Here, we theoretically investigate more advanced filter structures using similar principles. We tailor mode splitting in a resonator formed by two coupled Sagnac loop reflectors (CSLR) consisting of both FIR and IIR filter elements to achieve wavelength interleaving and non-blocking switching functions with flat-top spectral response. Other requirements for practical WDM systems including high extinction ratio (ER), low insertion loss (IL), low crosstalk and channel spacing (CS) meeting the ITU-T standards [16] are also considered in our design and achieved for the proposed device. These results highlight the strong potential of using CSLR resonators to achieve wavelength interleaving and non-blocking switching in WDM systems.

\section{Device configuration and operation principle}

Figure 1(a) shows the schematic of the proposed CSLR resonator, which consists of two parallel SLRs coupled to a top bus waveguide. The two SLRs are identical and each SLR is formed by a self-coupled SOI nanowire with a length of $L_{\text {SLR }}$. Here, $t_{1}$ and $\kappa_{1}$ are the field transmission and cross coupling coefficients of the directional couplers in the SLRs, respectively, while $t_{2}$ and $\kappa_{2}$ are the field transmission and cross coupling coefficients of the directional couplers formed by the SLRs and the top bus waveguide, respectively. $L_{i}(i=1,2)$ are the lengths of the top and bottom connecting waveguides between the two SLRs. For the SOI nanowire with a cross section of $500 \mathrm{~nm} \times 260 \mathrm{~nm}$, we use a waveguide group index for TE polarization of $n_{\mathrm{g}}=4.3350$ and a propagation loss of $\alpha=55 \mathrm{~m}^{-1}$ (i.e., $2.4 \mathrm{~dB} / \mathrm{cm}$ ) based on our fabricated devices [14, 15]. The CSLR resonator is equivalent to an MZI and an FP cavity when $t_{1}=1$ and $t_{2}=1$, respectively. When $t_{1} \neq 1$ and $t_{2} \neq 1$, it can be regarded as a hybrid filter consisting of both FIR and IIR filter elements. The mutual interaction between the FIR and IIR filter elements yields efficient coherent optical mode interference, which allows a flat-top spectral response for a device with only two SLRs. The transmission spectra of the CSLR resonator can be tailored by changing $t_{1}$ and $t_{2}$, which enables flexible spectral engineering with the CSLR resonator for many filtering functions.
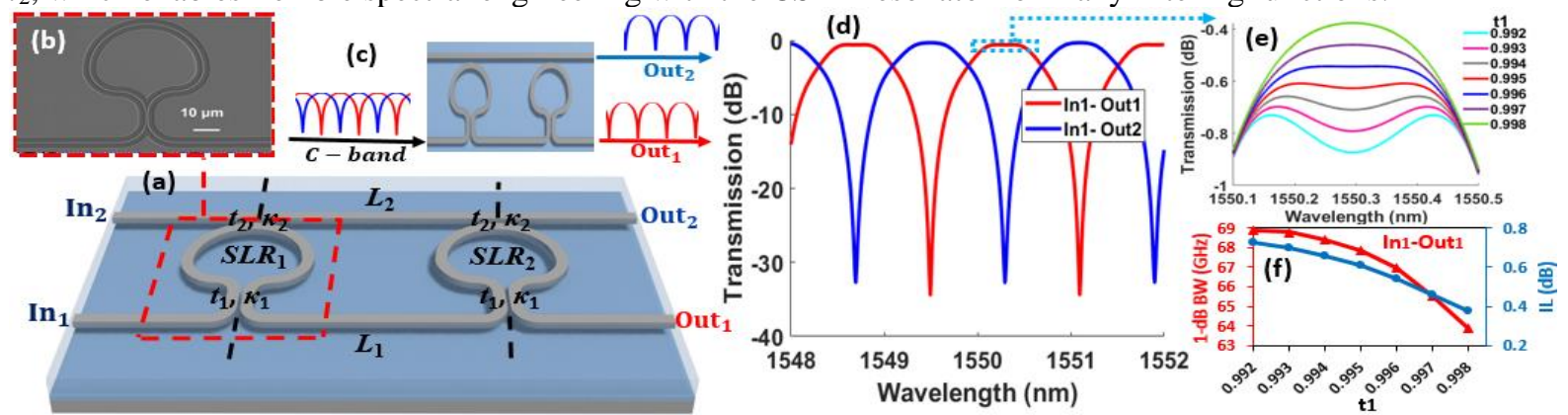

Fig. 1. (a) Schematic configuration of the proposed CSLR resonator. (b) SEM image of the fabricated SLR on an SOI wafer. (c) Interleaving operation based on the device in (a). (d) Output spectra at port Out $t_{1}$ and $\mathrm{Out}_{2}$ with input from port $\mathrm{In}_{1}$. (e) Filter shapes of the output spectra at port Out for different $t_{1}$. (f) $1-\mathrm{dB}$ bandwidth and insertion loss versus $t_{1}$. 
The scanning electron microscope (SEM) image of a fabricated SLR is shown in Fig. 1(b). The interleaving operation based on the CSLR resonator is shown in Fig. 1(c). Figure 1(d) shows the power transmission spectra calculated based on the scattering matrix method $[14,15]$ with input from port $\operatorname{In}_{1}$. The structural parameters $\left(L_{S L R 1,2}=L_{1,2}=346 \mu \mathrm{m}, t_{1}=0.995, t_{2}=0.707\right)$ are designed for achieving a CS of about $100 \mathrm{GHz}$ in C band to meet the ITU-T spectral grid standards G694.1. Figure 1(e) shows the filter shapes of the output spectra at port Out1 for different $t_{1}$. The calculated 1-dB bandwidth (BW) and IL as functions of $t_{1}$ are depicted in Fig. 1(f). Table 1 compares the $0.5-\mathrm{dB}$ and $1-\mathrm{dB}$ BWs, ERs and ILs of the MZI (when $t_{1}=1$ ) and the CSLR resonators with input from different ports $\left(\mathrm{In}_{1}\right.$ and $\left.\mathrm{In}_{2}\right)$. As compared with the MZI, the CSLR resonator shows a significantly improved top flatness at the expense of slightly reduced ERs and ILs within reasonable ranges.

Table 1. Performance comparison of the interleavers based on MZI and CSLR resonators.

\begin{tabular}{|c|c|c|c|c|c|c|}
\hline & \multicolumn{2}{|c|}{$\operatorname{MZI}\left(t_{1}=1\right)$} & \multicolumn{2}{|c|}{$\begin{array}{l}\text { CSLR resonator } \\
\text { with input port from } \mathrm{In}_{1}\end{array}$} & \multicolumn{2}{|c|}{$\begin{array}{c}\text { CSLR resonator } \\
\text { with input port from } \mathrm{In}_{2}\end{array}$} \\
\hline & Out $_{1}$ & $\mathrm{Out}_{2}$ & Out $_{I}$ & $\mathrm{Out}_{2}$ & Out $_{I}$ & $\mathrm{Out}_{2}$ \\
\hline 0.5-dB BW (GHz) & 42.7688 & 42.7852 & 56.1612 & 44.4834 & 46.6557 & 45.9546 \\
\hline 1-dB BW (GHz) & 59.9887 & 59.9767 & 67.8828 & 60.8639 & 60.9014 & 60.6391 \\
\hline ER (dB) & 45.9179 & 46.4525 & 33.7906 & 32.3885 & 32.3885 & 30.9091 \\
\hline IL (dB) & 0.2065 & 0.1652 & 0.6083 & 0.3341 & 0.3341 & 0.2068 \\
\hline CS (GHz) & \multicolumn{2}{|c|}{100.0053} & \multicolumn{2}{|c|}{100.0053} & \multicolumn{2}{|c|}{100.0053} \\
\hline
\end{tabular}

We also design $2 \times 2$ non-blocking switching unit based on the CSLR resonator. Figure 2(a) shows the zoomin view of Fig. 1(d). For comparison, the transmission spectra from $\mathrm{In}_{2}$ to $\mathrm{Out}_{1}$ and $\mathrm{Out}_{2}$ in the same wavelength range are depicted in Fig. 2(b). Two wavelength channels centered at $\lambda_{1}=1549.4938 \mathrm{~nm}$ and $\lambda_{2}=1550.29456$ $\mathrm{nm}$ are selected for the operation of the cross (Fig. 2(c)) and bar (Fig. 2(d)) states, respectively. When $\lambda_{1}$ is red shifted to $\lambda_{2}$ via thermo-optic effect, the switching unit changes from cross state to bar state. The calculated ERs, ILs and crosstalk are shown in Table 2. As can be seen, flat-top spectral response with high ERs, low ILs and low crosstalk are achieved. When input port is changed to $\mathrm{In}_{2}$, the wavelength channels for the cross and bar states remain unchanged, i.e., $\lambda_{1}=\lambda_{1}{ }^{\prime}$ and $\lambda_{2}=\lambda_{2}$ '.
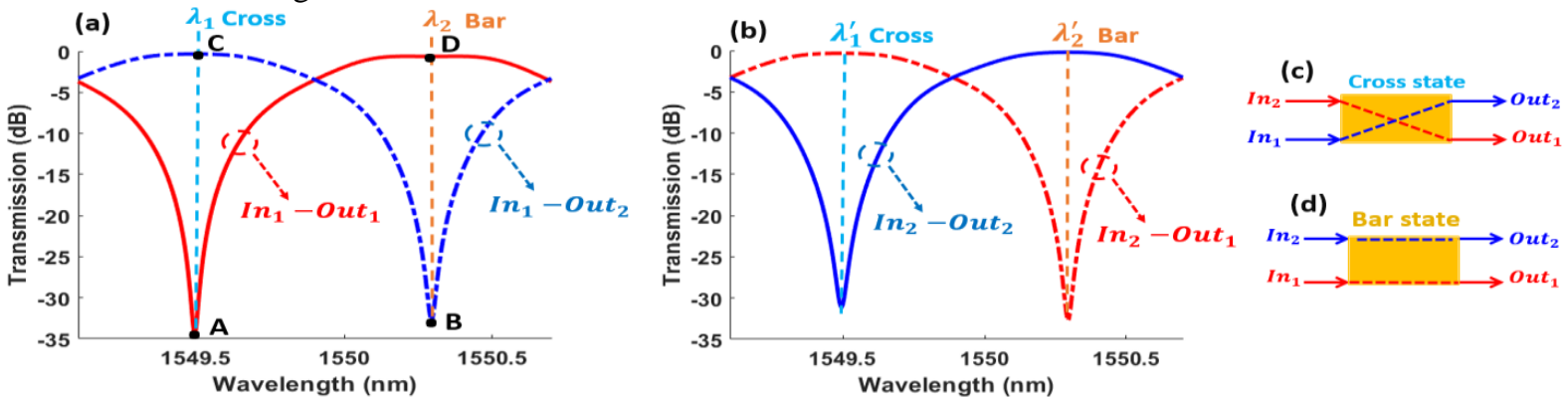

Fig. 2. (a) Zoom-in view of Fig. 1(d) in the wavelength range of $1549.1 \mathrm{~nm}-1550.7 \mathrm{~nm}$. (b) Output spectra at port Out ${ }_{1}$ and Out ${ }_{2}$ with input only from port $\mathrm{In}_{2}$. (c)-(d) Schematics showing the cross and bar states of the non-blocking switching unit, respectively.

Table 2. Performance of the non-blocking switching unit based on CSLR resonator.

\begin{tabular}{|l|c|c|}
\hline Operation state & Cross & Bar \\
\hline Operation wavelength $(\mathbf{n m})$ & $\lambda_{1}=\lambda_{1}^{\prime}=1549.4938$ & $\lambda_{2}=\lambda_{2}^{\prime}=1550.2945$ \\
\hline Extinction ratio $(\mathbf{d B})$ & $P_{C}-P_{B}=32.3885$ & $P_{D^{\prime}}-P_{A}=33.7906$ \\
\hline Crosstalk $(\mathbf{d B})$ & $P_{A}-P_{C}=-34.0669$ & $P_{B}-P_{D}=-32.1142$ \\
\hline Insertion loss $(\mathbf{d B})$ & 0.3341 & 0.6083 \\
\hline
\end{tabular}

\section{Conclusions}

We theoretically investigate wavelength interleaving and non-blocking switching based on integrated CSLR resonator. Flat-top spectral responses are achieved by tailoring mode splitting in the CSLR resonator.

\section{References}

[1] S. Cao, et al., "Interleaver technology: comparisons and applications requirements," J. Lightwave Technol., 22(1), $281,2004$.

[2] J. Wu, et al., "Non-blocking $2 \times 2$ switching unit based on nested silicon microring resonators with high extinction ratios and low crosstalks," Chin. Sci. Bull. 59, 2702, 2014.

[3] C. Gunn, "CMOS photonics for high-speed interconnects," IEEE Micro, 26(2), 58, 2006.

[4] D. J. Moss, R. Morandotti, A. L. Gaeta, and M. Lipson, "New CMOS-compatible platforms based on silicon nitride and Hydex for nonlinear optics," Nat. Photonics 7, 597-607 (2013).

[5] S. Lai, et al., "Compact silicon photonic interleaver based on a self-coupled optical waveguide," Appl Opt., 55(27), 7550, 2016.

[6] N. Zhou, et al., "Reconfigurable and tunable compact comb filter and (de)interleaver on silicon platform," Opt. Exp., 26(4), 4358, 2018.

[7] R. Soref, et al., "Multiple-Sagnac-loop Mach-Zehnder interferometer for wavelength interleaving, thermo-optical switching and matched filter," J. Lightwave Technol., 36(22), 5254, 2018.

[8] L. Luo, et al., "High bandwidth on-chip silicon photonic interleaver," Opt. Exp., 18(22), 23079, 2010. [8] J. Wu, et al., "Compact onchip $1 \times 2$ wavelength selective switch based on silicon microring resonator with nested pairs of subrings,” Photon. Res. 3(1), 9, 2015 .

[9] D.J. Moss et al.,"Tunable dispersion and dispersion slope compensators for 10 Gb/s using all-pass multicavity etalons", IEEE Photonics Technology Letters, vol. 15, no. (5), 730-732 (2003). DOI: 10.1109/LPT.2003.809921. 
[10] D.J. Moss et al., "Multichannel tunable dispersion compensation using all-pass multicavity etalons", paper TuT2 Optical Fiber Communications Conference, Anaheim (2002). Postconference Technical Digest (IEEE Cat. No.02CH37339). Opt Soc. America. Part vol.1, 2002, pp. 132-3. Washington, DC, USA.

[11] D.J. Moss et al.,"Tunable dispersion compensation at $10 \mathrm{~Gb} / \mathrm{s}$ and $40 \mathrm{~Gb} / \mathrm{s}$ using multicavity all-pass etalons", Optical Fiber Communications Conference (OFC) paper TuD1, page 162, Atlanta, GA, March (2003). Post-conference Digest (IEEE Cat. No.03CH37403). Opt. Soc. America. Part vol.1, 2003, pp. 162-3. Washington, DC, USA.

[12] L. Lunardi et al.,"Tunable dispersion compensators based on multi-cavity all-pass etalons for 40Gb/s systems", Journal of Lightwave Technology, vol. 20, no. 12, 2136 (2002). DOI: 10.1109/JLT.2002.806768.

[13] L.M. Lunardi et al., "An Etalon-Based Tunable Dispersion Compensator Device for 40-Gbit/s Applications”, European Conf. on Optical Communications, Paper 5.4.6 Copenhagen, Sept. (2002). vol. 2, 2002, Piscataway, NJ, USA. Print ISBN: 87-90974-63-8.

[14] J. Wu, et al., "Advanced photonic filters based on cascaded Sagnac loop reflector resonators in SOI nanowires," APL Photon., 3(4), $046102,2018$.

[15] J. Wu, et al., "Micro-ring resonator quality factor enhancement via an integrated Fabry-Perot cavity," APL Photon., 2(5), $056103,2017$.

[16] http://handle.itu.int/11.1002/1000/11482 